\title{
OPTIMIZED ULTRA-WIDEBAND RADIATION OF DIPOLE ANTENNAS WITH TRIANGLE DRIVING PULSES
}

\author{
Anatoliy O. Boryssenko*, Daniel H. Schaubert*
}

\section{INTRODUCTION}

This paper addresses optimization of transient radiation from dipole antennas in terms of minimized pulse extent in time or flat power spectral density. The former is a typical requirement for high-range-resolution radars while the latter can meet the Federal Communication Commission (FCC) regulations on spectrum usage. Recent needs and advances in ultra-wideband (UWB) signal processing, target identification, remote and subsurface sensing, reach-frequency tagging, secure communication, and others stimulate a variety of diverse studies in transient and broadband electromagnetics, including pulsed antennas. These generic problems have been widely explored through full-wave numerical analysis, theoretical and asymptotic approaches, nonlinear optimization methods combined with full-wave electromagnetic solvers, the E- and K-pulse signal processing techniques, and other fashions.

Previous work often involves antennas of complicated geometry/structure and/or pulses of complex shapes to drive the antennas' currents. The work reported here is based on three guiding requirements:

1. Geometrically simple antennas like thin-wire linear dipole, flat dipole, bow-tie radiator and conical dipole.

2. Simple triangle-like pulse shapes of voltage source to reduce the complexity of driving electronics.

3. Operation below the first parallel resonance of the antenna so the radiator is not large in size.

The optimal signals for driving of the dipole antennas are realized here in numerical simulation through full-wave method of moment (MoM) numerical analysis, and combined MoM simulation and nonlinear optimization. Results of the electromagnetic analysis are supported with circuitry modeling in both time and frequency domains also.

${ }^{*}$ Antenna Laboratory, University of Massachusetts, Amherst, MA, 01003, USA 


\section{PROBLEM FORMULATION}

It is accepted ${ }^{1,2,3}$ that conventional wideband antennas like dipoles often do not perform well under wideband excitation because of their dispersive characteristics. In this work, we propose some novel operational modes of those antennas, which enable controlled UWB behavior by using their inherent dispersive properties. The problem can be solved with the help of specially constructed waveforms of driving signals that are matched in some sense with the antennas. The primary intent here is to achieve some specific performance criteria including the radiation of temporally short and/or broad bandwidth pulses. Particular attention is given in this work to transmitting antennas of simple geometries, like center-fed thin-wire and flat dipoles, Figure 1.

It is known ${ }^{1,2}$ that the simplest solution to support short radiated pulses is using an electrically very short antenna whose radiation is proportional to the time derivative of the driving waveform. Resistively loaded antennas ${ }^{4}$ can be used to reduce unwanted reflection and associated pulse distortions. However, electrically small antennas and resistive loading are not always applicable due to poor radiation efficiency. Other related studies in the transient antennas involve structures of composite geometry and/or complex waveform for excitation such as log-periodic antennas with chirp pulse 5 , Vshape dipoles, TEM-horns, tapered-slot antennas, reflectors, lenses, and others ${ }^{3}$. For the purpose of our project, forward protruding end-fire radiators do not meet the requirement for small volume.

Moreover, the FCC regulates the EM spectrum for UWB devices. Thus, besides flatness, simplicity in geometry, and efficiency in radiation, waveform/spectrum control is required that has to be consistent with realistic solutions for the driving electronics. Various methods based mainly on signal processing like E- and K-pulse techniques are not well suited for direct implementation in antennas' hardware and left beyond the scope of our research. However, we employ widely methods of optimization combined with full-wave numerical analysis ${ }^{6}$ for improving the transient characteristics in antennas.

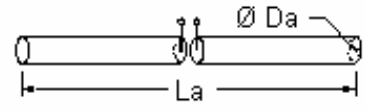

(a)

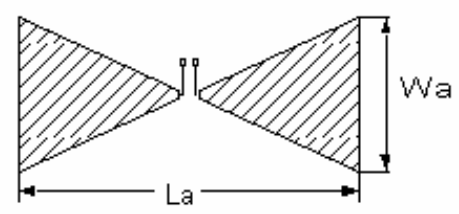

(c)

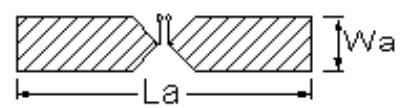

(b)

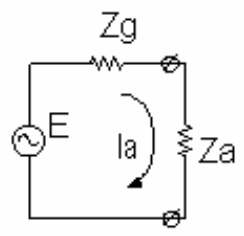

(d)

Figure 1. Center-fed dipole antennas explored for optimal radiation: (a) thin-wire dipole; (b) flat dipole; (c) bow-tie dipole; (d) generalized equivalent electrical circuit. 


\section{ANALYSIS THROUGH TIME-DOMAIN INTEGRAL-EQUATION SOLVER}

Full-wave analysis of the antennas in Figure 1 is performed by using a time-domain electric integral equation solver ${ }^{7}$ that we developed. It simulates, through marching in time, 3-D electromagnetic problems with thin-wire and surface-patched elements. Usually, when a thin wire dipole is center-fed with an arbitrary exciting signal, the terminal current in the antenna has long oscillations in late time because the antenna has high-Q resonances. Figure 2a shows this result for a sinusoidal burst with Gauss pulse envelope. The spectral maximums for the current correspond to the resonant frequencies covered by the spectrum of the exciting waveform. Surprisingly, a simple asymmetric triangle pulse applied to the same antenna extinguishes the late-time oscillations, Figure $2 \mathrm{~b}$, when the appropriate pulse length and ratio of rise-to-fall time is provided. The antenna current resembles a single sinusoidal cycle while the triangular source is applied and a low-amplitude ringing during source-free interval. The power spectrum of the triangular pulse is mostly below the first parallel resonance of the 0.5 -meter antenna. The spectral power density of the terminal current is relatively broad and flat and centered near $150 \mathrm{MHz}$. The source is "matched" to the antenna in the sense that its power spectral density compensates for the low-frequency response of the antenna and excites the first series resonance sufficiently to broaden and flatten the current spectrum.

Figure 3 illustrates computed far-field radiation patterns in time-domain for the same cases as in Figure 2. For the optimal triangle pulse, Figure 2b, the radiated waveform has dominant sinusoidal-like triplet with low oscillations in source-free interval. Optimizations may be performed on the radiated field or the terminal current. Hereafter, we will illustrate the antennas' driving/terminal current.
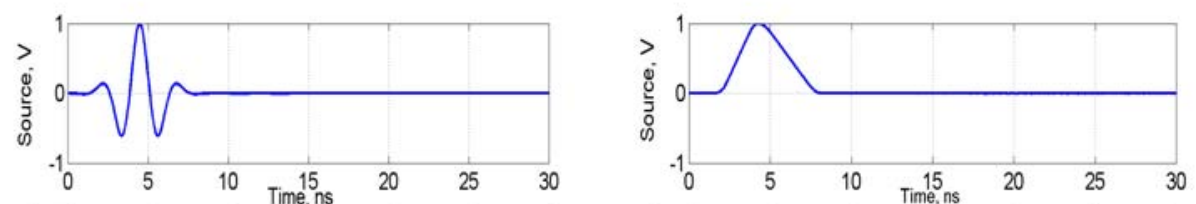

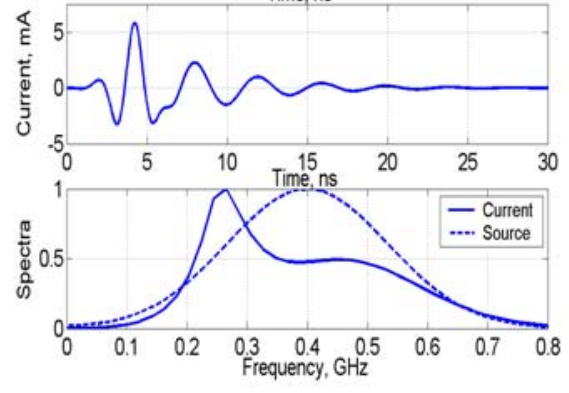

(a)

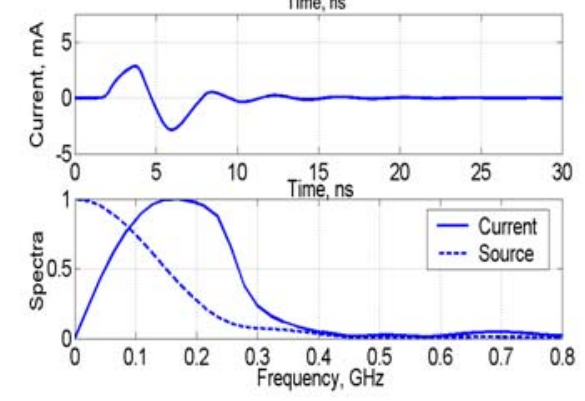

(b)

Figure 2. Transient excitation of the center-fed 0.5-meter thin wire dipole in transmit mode by: (a) Gauss pulse: (b) optimized triangle pulse. 


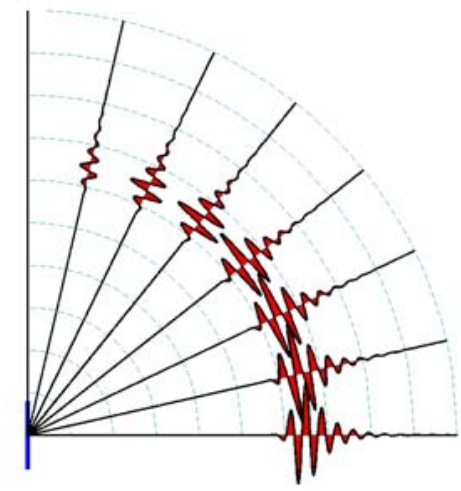

(a)

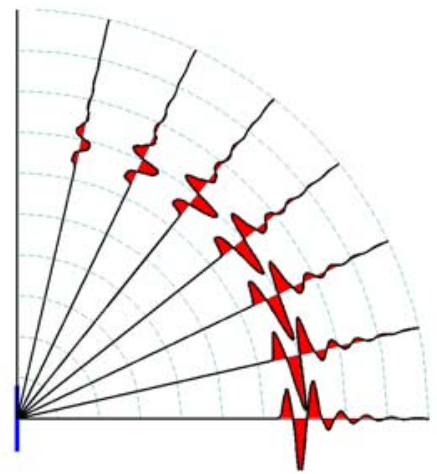

(b)

Figure 3. Far-field radiation pattern for (a) Gauss and (b) triangle pulses.

The shape and spectrum of the optimal triangle pulse are shown in Figure 4. In fact, the triangle shape of the pulse has been discovered by chance during extensive calculations with the MoM code. Similar waveforms can be found for all of linear antennas in Figure 1, but only for the thin wire dipoles the ratio of rise-to-fall time is amazingly close to the golden section number, Figure 4a. However, optimization techniques combined with MoM analysis ${ }^{6}$ can be employed effectively to optimize transient behavior of many antennas in the same way. For example, Figure 5 demonstrates this approach applied to the flat dipole. Two principal cases are seen in Figure 5. The first one corresponds to optimization with a goal function to support welllocalized spectrum, Figure 5a, while optimization in the second case, Figure 5b, is aimed at obtaining temporally short pulse but by some price of small spreading in the spectrum.

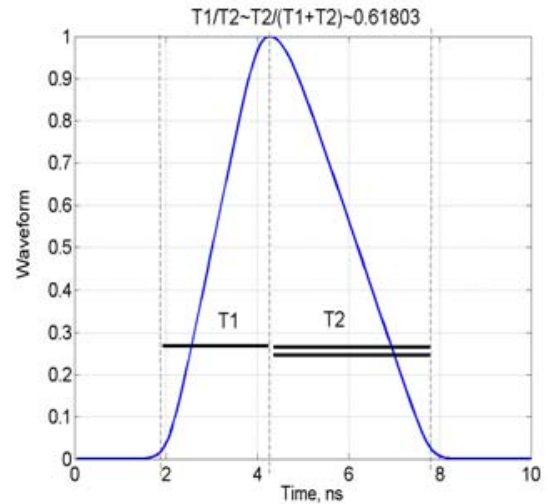

(a)

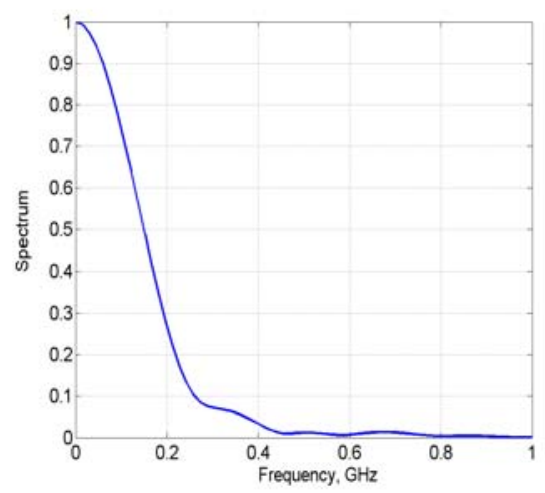

(b)

Figure 4. An optimal triangular pulse for the thin-wire 0.5 -m dipole: (a) pulse waveform with rise-to-fall ratio equals about to the golden section number; (c) power spectrum. 

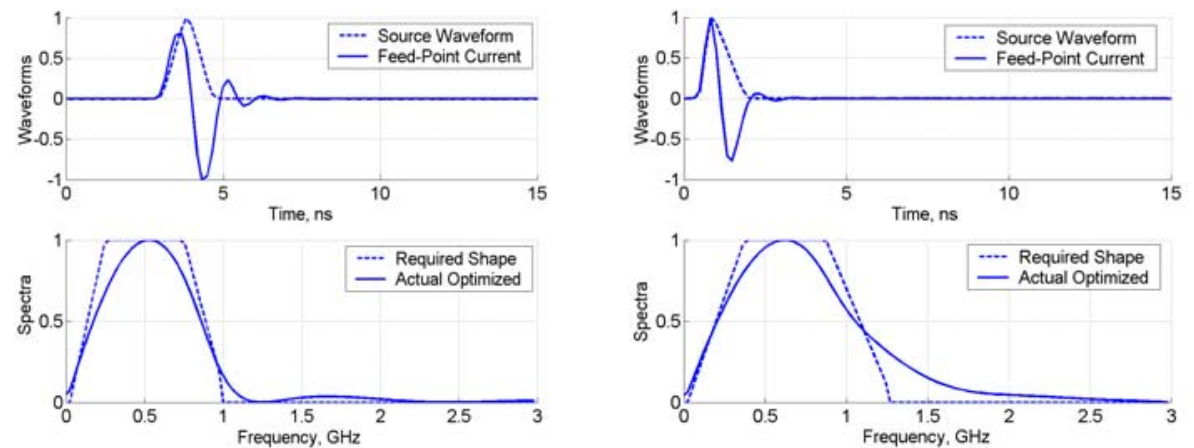

(a)

(b)

Figure 5. Some optimization for center-fed bow-tie dipole of $10-\mathrm{cm}$ length and $5-\mathrm{cm}$ width to provide: (a) localized spectrum; (b) shortest pulse duration.

When optimizing for short time duration, greater than $95 \%$ of the pulse response energy belongs to sine wave doublet, which coincides with the duration of the triangular voltage pulse applied to the antenna. When optimizing for flat spectrum, a typical 4:1 half-power bandwidth is achieved with nearly all of the power at frequencies below the first parallel resonance of the dipole. The optimization procedure can be repeated for different goal functions tracking good localization for the antenna pulsed response in spectrum or in time or in a mixed mode.

A simple phenomenological interpretation in terms of radiation physics of transient antennas helps to explain the found effect of the triangle pulse. Obviously, the driving waveform delivers the pulsed energy to the antenna. Due to finite lengths of both the pulse and antenna this energy comes into the antenna consecutively through its terminals and propagates as a current/charge wave in it. The portion of the wave belonging to the front of the pulse will be reflected from the open edges of the antenna and returned back to the feed point. Nearly at the time when the reflection happens the second portion of the driving pulse with falling edge comes into the antenna through the terminal and annihilates the back-reflected wave because of phase inversion after reflection from the antenna's open edges. In the end, the current exists in the antenna when the source pulse is present there only, and there is little ringing. The pulse is asymmetric to compensate for dispersion in the antenna.

\section{ANALYSIS BASED ON EQUIVALENT ELECTRICAL CIRCUITS}

Since we use simple antennas that are electrically small, the radiating currents of the antenna (or the fields if we choose to consider them) are related to the source voltage by a simple equivalent circuit. We can optimize antenna current performance by considering only the impedance of the antenna and the voltage generator. Thus, this problem of antenna optimizing can be reduced to the simpler problem of circuit optimization. 
The equivalent circuit of any antenna can be defined through the samples of frequency-domain data on the antenna input impedance by least-square fitting of these actual full-wave data and those from the equivalent circuit. For example, Figure 6 shows the equivalent circuit for bow-tie dipole of the specified geometry and demonstrates excellent agreement between both the models over a very broad bandwidth. This simple equivalent circuit can be used to explore the behavior of the driving electronics over a frequency range broader than the antenna's operating bandwidth.

A simple mathematical model, based on the equivalent circuit in Figure 1d, can be utilized for synthesis of an optimized pulse. A source waveform $E(t)$ that provides at the antenna terminals some required spectral properties for the current $I(\omega)$ can be obtained from:

$$
E(t)=F_{-}\{I(\omega) \cdot Z(\omega)\}
$$

where $F_{-}$- inverse Fourier transform operator; $I(\omega)$ - required current response, approximated through polynomial or error-function for flat spectrum density and linear phase; $Z(\omega)$ - input impedance of antenna like that presented in Figure 6.

Figure 7 contains typical results for the antenna in Figure 6. Four cases of increasing bandwidth are presented. If the frequency range of the signal is approximately $0.2-0.7$ of the first parallel resonant frequency of the dipole antenna, Figure 7a, a relatively simple voltage waveform compensates for the variation of the antenna's impedance function, resulting in approximately uniform power spectral density. However, if the signal's frequency range includes the first parallel resonance, Figure $7 \mathrm{~d}$, or close to it, Figure $7 \mathrm{c}$, the voltage waveform must be more complicated and contains a large power density near the resonance to offset the reduced response in this frequency range. The intermediate case, Figure $7 \mathrm{~b}$, confirms the above observations on the significance of proper choice of bandwidth with respect to the resonant frequency.
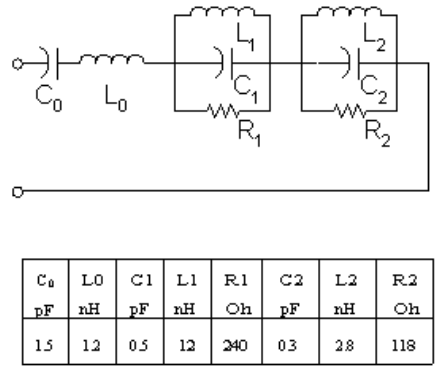

(a)
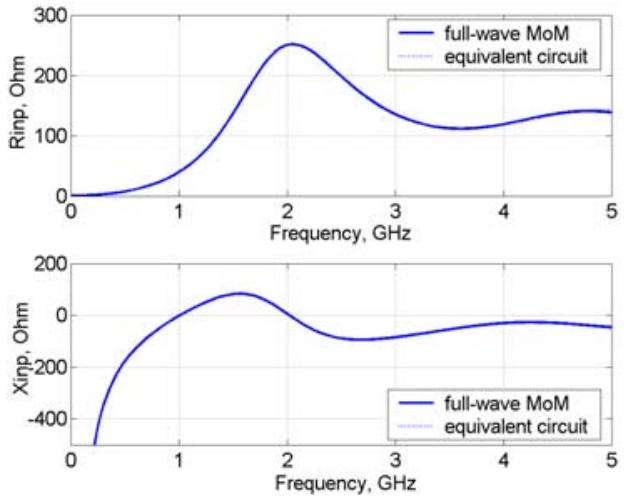

(b)

Figure 6. Bow-tie dipole of 10-cm length and 5-cm width: (a) equivalent circuit; (c) input impedance through the full-wave MoM analysis and the equivalent circuitry. 
The antenna current responses in Figure 7 demonstrate several other features worth mentioning:

1. The most useful bandwidth of the antenna ranges up to $0.7-0.8$ of its first arallel resonance.

2. Relatively simple voltage waveforms yield moderately well localized current in both frequency and time domains.

3. Bandwidths in excess of $6: 1(-3 \mathrm{~dB}$ level) can be supported with simple voltage pulses suitable for implementation through driving electronics.

4. Localization of the antenna driving current in time is inversely proportional to the achieved bandwidth.

5. Size scaling would maintain simplicity of voltage waveform while increasing operating frequency.
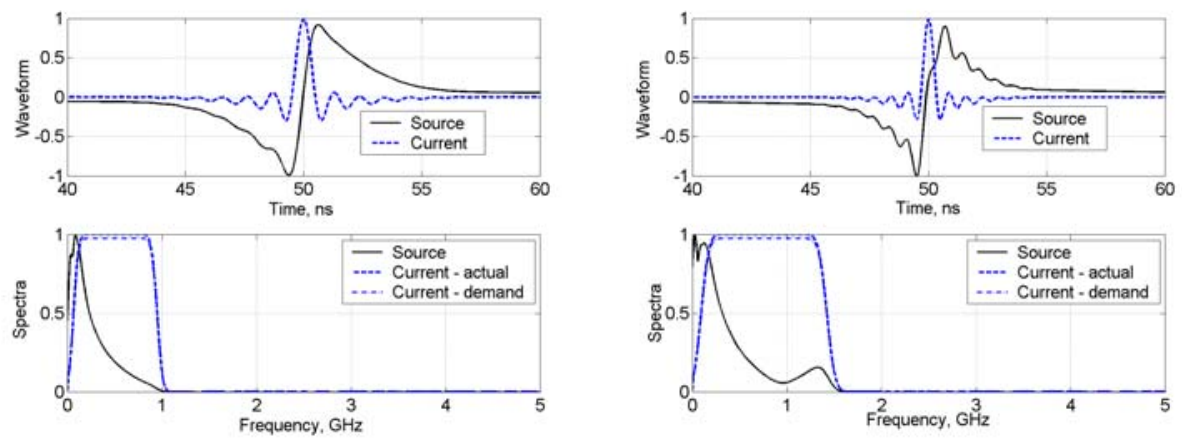

(a)

(b)
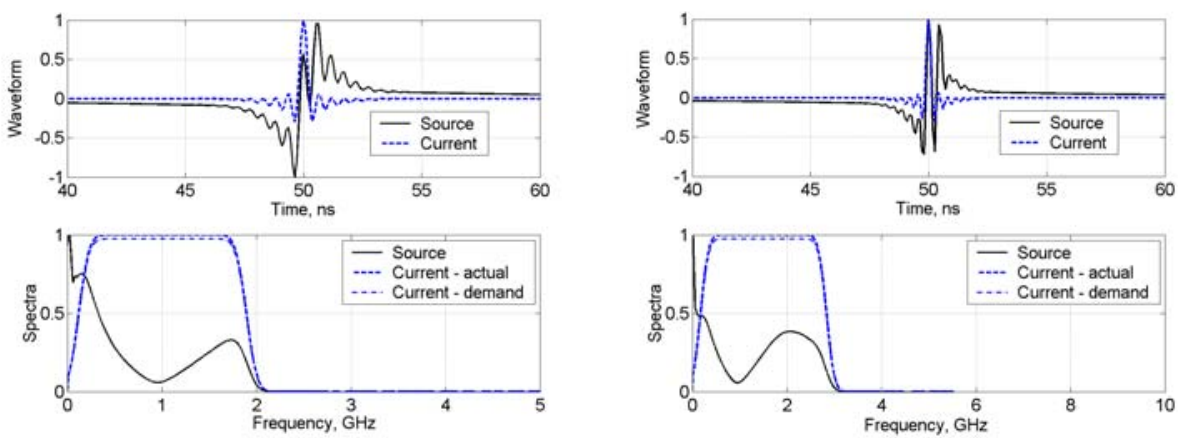

(c)

(d)

Figure 7. Bow-tie dipole of $10-\mathrm{cm}$ length and 5 - $\mathrm{cm}$ width with optimizations for bandwidth of about: (a) 0.2 $0.8 \mathrm{GHz}$; (b) $0.2-1.2 \mathrm{GHz}$; (c) $0.2-1.7 \mathrm{GHz}$; (d) $0.4-2.5 \mathrm{GHz}$. 


\section{CONCLUSIONS AND DIRECTIONS OF FUTURE WORK}

Some solutions to support high performance pulse radiation modes in the dipole-like antennas are presented. Two types of waveforms for pulse driving in the antennas are revealed; first, the triangle pulse with DC components in spectrum and odd response function in time and, second, the triangle-like doublet with zero DC and even response function in time. In both cases, simple electronic circuits can generate the voltage driving waveform. In general, the methods presented here yield transient waveforms to meet specific performance criteria for band limited antennas.

The demonstrated analysis and its results are directly applicable to control the shapes of pulses and their spectra for the dipoles, and maybe other antennas, by constructing of the necessary driving signals. For example, the cases shown here exhibit a dominant early-time response (more than $95 \%$ of the pulse energy) and very low late-time tails. At the same time, much of energy of antenna response can be effectively localized in a specific frequency band below the first parallel resonance in the antennas. Because antennas of nearly resonance length without resistive loading are employed, radiation efficiency is high and the antennas are of moderate size. Our current and further efforts are aimed at looking for new types of exciting waveforms to be employed in time-hoping UWB wireless systems and realized through CMOS integral circuitry. The actual antenna-driver configuration becomes extremely important to enhance overall performance of the UWB system.

\section{ACKNOWLEDGMENTS}

This research was supported by the MURI grant DAAD19-01-1-0477, "Short-Range Ultra-Wideband Systems."

\section{REFERENCES}

1. G. Franceschetti, C.H. Papas, Pulsed Antennas, IEEE Trans. Ant. Propag. 22(5), pp. 651-661 (1974).

2. H. F. Harmuth, Antennas and Waveguides for Nonsinusoidal Waves (New York, Academic Press, 1984).

3. C. E. Baum, E. G. Farr, Impulsive Radiating Antennas, in: Ultra-Wideband Short-Pulse Electromagnetics, edited by H. Bertoni et al. (Plenum, New York, 1993), pp. 139-147.

4. T. T. Wu, R. W. P. King, The Cylindrical Antenna with Nonreflecting Resistive Loading, IEEE Trans. Ant. Propagat. 13(3), pp. 369-373 (1965).

5. P. S. Excell, A. D. Tinniswood, R.W. Clarke, An Independently Fed Log-Periodic Antenna for Directed Pulsed Radiation, IEEE Trans. Ant. Propagat. 41(4), pp. 344-349 (1999).

6. J. R. Bayard, D. H. Schaubert, Target Identification Using Optimization Technique, IEEE Trans. Ant. Propagat. 38(4), pp. 450-456 (1990).

7. A. O. Boryssenko, D. H. Schaubert, Time-Domain Integral-Equation-Based Solver for Transient and Broadband Problems in Electromagnetics, in: This publication. 